\title{
Radical copper oxidases, one electron at a time
}

\author{
James W. Whittaker and Mei M. Whittaker
}

Oregon Graduate Institute of Science and Technology, Portland, Oregon 97291-1000 U.S.A.

\begin{abstract}
Radical copper oxidases (including the fungal enzymes galactose oxidase and glyoxal oxidase) are emerging as an important family of metalloenzymes based on the free radical-coupled copper catalytic motif. The active sites of these enzymes combine a redox active copper ion with a stable protein free radical, forming a two-electron redox unit capable of oxidizing a variety of alcohols and aldehydes with reduction of dioxygen to hydrogen peroxide. This active site is remarkable in the extent to which the ligands participate in catalysis. One of the tyrosine residues dissociates from the metal center and abstracts a proton to activate substrate for oxidation, while a second tyrosine (post-translationally modified to form a tyrosine-cysteine dimer) serves as the free radical redox site. Computational studies of the substituted phenoxyl indicates that in the ground state the majority of the unpaired electron is localized on the phenoxyl oxygen and thioether sulfur atoms, accounting for the special properties of this site. Isotope kinetics have been investigated for the substrate oxidation half-reaction indicating a dramatic isotope effect $\left(k_{\mathrm{H}} / k_{\mathrm{D}} \sim 20\right)$ consistent with homolytic cleavage of the methylene $\mathrm{C}-\mathrm{H}$ bond and hydrogen atom transfer to the phenoxyl in the transition state.
\end{abstract}

\section{INTRODUCTION}

Radical copper oxidases [1] combine two distinct redox centers (one a metal ion, the other a stable protein free radicitl) into an efficient catalytic unit, a free-radical coupled copper complex. In the fungal metalloenzymes galactose oxidase (GAO, from Fusarium sp.) [1-5] and glyoxal oxidase (GLX, from $P$ hanerochaete chrysosporium) [6,7] this radical-Cu motif performs two-electron oxidation of simple alcohols and aldehydes with reduction of $\mathrm{O}_{2}$ to hydrogen peroxide:

$\begin{array}{ll}\text { GAO: } & \mathrm{RCH}_{2} \mathrm{OH}+\mathrm{O}_{2} \rightarrow \mathrm{RCHO}+\mathrm{H}_{2} \mathrm{O}_{2} \\ \text { GLX: } & \mathrm{RCHO}+\mathrm{O}_{2} \rightarrow \mathrm{RCO}_{2} \mathrm{H}+\mathrm{H}_{2} \mathrm{O}_{2}\end{array}$

Recently a third member of this family, an extracellular oxidase from the prokaryote Stigmatella aurantiaca, has been identified by sequence homology [8]. In addition to conservation of the metal ligands, all three proteins preserve a special framework, the superbarrel fold, which supports the active site.

Each of these enzymes appears to have a distinct biological function. Galactose oxidase is thought to have a bacteriostatic role, allowing the fungus to compete more effectively for nutrients, thus paralleling the function of the fungal flavoenzyme glucose oxidase. In contrast, glyoxal oxidase expression is coordinated with a suite of peroxidases involved in lignin degradation, including lignin peroxidase and manganese peroxidase. Glyoxal oxidase supplies the peroxide co-substrate for ligninolytic peroxidase reactions and is thus a key player in an environmentally important process [6]. The homolog from Stigmatella is less well characterized but is thought to have a role in fruiting body formation [9].

In spite of their names, both galactose and glyoxal oxidases exhibit an unusually broad specificity for the reducing substrate $[2,3,6]$. Although each preferentially processes primary alcohols or aldehydes, respectively, the group $R$ in equations (1) and (2) can represent a wide range of structures. The biologically important product in both of these reactions appears to be hydrogen peroxide, and the broad specificity allows a variety of small reductants to support turnover. Both enzymes exhibit very rapid rates for both substrate oxidation and $\mathrm{O}_{2}$ reduction half-reactions, apparently reflecting a trade-off favoring increased catalytic speed at the expense of selectivity. 


\section{ACTIVE SITE STRUCTURE}

The structure of galactose oxidase has been solved at $1.7 \AA$ resolution [10], revealing the basic geometric details of the enzyme active site. As predicted by spectroscopy [11], the copper site is unusual in having tyrosine residues involved in metal binding. The $\mathrm{Cu}$ ion is bound by two tyrosine phenolates (Y272,Y495) and two histidine imidazoles (H496,H581), with a solvent molecule completing the distorted pyramidal coordination polyhedron. One of the tyrosines is covalently crosslinked to a cysteine residue forming a new amino acid, a tyrosine-cysteine dimer, that has been identified as the radical-forming site in the protein by spectroscopic and biochemical investigations [1]. A tryptophan indole ring lying over the thioether crosslink may serve a protective role, shielding the thioether sulfur from reaction with the product peroxide. The low symmetry protein environment for the metal center places the solvent (water) in the axial position with a long metal-ligand bond distance characteristic of a weakly interacting ligand. The shortest bond distance is associated with the strongly bound Tyr-Cys dimer (Y272) phenolate while the M-L bond distance of the unmodified tyrosine (Y495) is somewhat ambiguous in terms of coordination state (see below).

The active site of glyoxal oxidase is spectroscopically nearly identical to that of galactose oxidase, with a coordination shell including both an unmodified tyrosinate and a Tyr-Cys dimer in addition to a bis-histidine ligand complement [7]. Sequence alignments predict that the tryptophan overlying the Tyr-Cys site in galactose oxidase is replaced by histidine in glyoxal oxidase [8], which may account for differences in active site properties [7] including redox stability, $\mathrm{pH}$ sensitivity, anion binding reactivity, and the significantly different redox potentials for the two radical-forming sites (galactose oxidase, $E^{\circ}=0.45 \mathrm{~V} v \mathrm{~s}$. NHE; glyoxal oxidase, $\left.E^{\circ}=0.66 \mathrm{~V} v s . \mathrm{NHE}[7]\right)$.

The details of coordination geometry can be expected to have important consequences in controlling the reactivity of tyrosinate ligands and determining their role in metalloenzyme catalysis. Tyrosinate coordination can be characterized by two angular parameters $(\theta$, the C-O-M phenolate bond angle and $\tau$, the ring torsion or dihedral angle) in addition to the M-O bond distance, $r$ (Fig. 2). The limiting 'in-plane' $\left(\tau=0^{\circ}\right)$ and 'perpendicular' $\left(\tau=90^{\circ}\right)$ coordination modes will each lead to distinct perturbations of both the metal and ligand electronic structures. In-plane coordination leads to strong sigma (donor) overlap between the nonbonding phenolate oxygen $2 \mathrm{p}_{\mathrm{x}}$ valence orbitals and the metal ion $3 \mathrm{~d}$ shell, while perpendicular coordination results in sigma interactions involving the phenolate $\pi$ system $\left(\mathrm{O} 2 \mathrm{p}_{\mathrm{z}}\right)$. Experimental tyrosinate coordination modes for two protein $\mathrm{Cu}$ complexes and two synthetic model compounds are listed in Table 1 . The preference for $\mathrm{p}_{\mathrm{z}}$ - over $\mathrm{p}_{\mathrm{x}}$-coordination in proteins may be important in controlling reactivity of tyrosine phenolates (in both acid/base and redox reactions) and is a feature that needs to be addressed in synthetic modelling studies. The strong inclination toward perpendicular orientation of the Y272 ring system in galactose oxidase is particularly interesting in terms of the $\pi$ localization of the phenoxyl radical in the active enzyme (see below).

Exogenous ligands displace the coordinated solvent in the galactose oxidase active site, binding directly to the active site $\mathrm{Cu}$ ion. Crystallography suggests the effects of exogenous ligand binding has relatively minor effect on active site structure, and virtually no change is detected in the positions of the endogenous protein

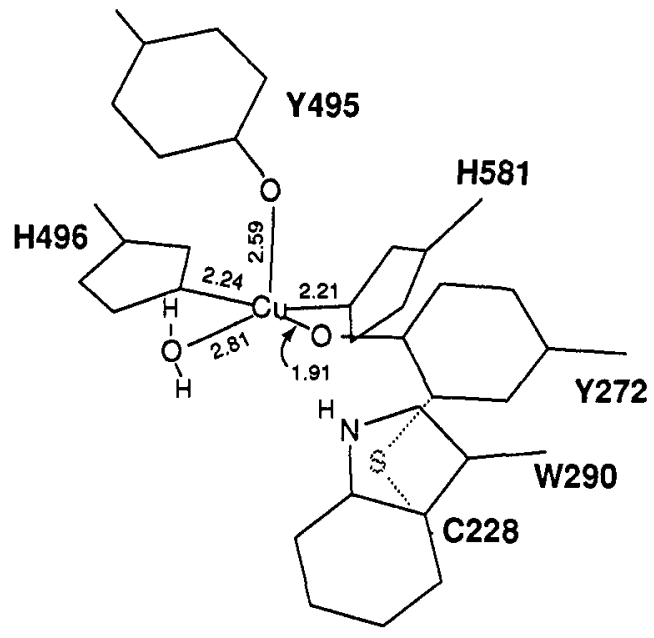

Fig. 1. Galactose oxidase active site structure (based on data in Ref. (10)).

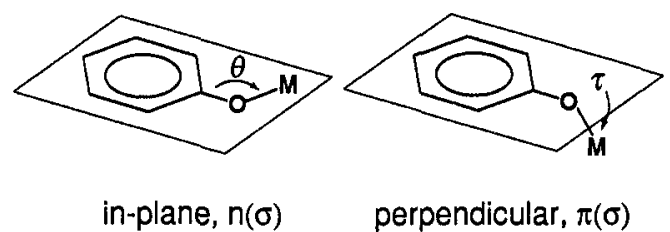

Fig. 2. Limiting coordination modes for tyrosinate ligands.

TABLE 1. Ligation parameters for $\mathrm{Cu}$ phenolate complexes.

\begin{tabular}{|c|c|c|c|c|c|}
\hline \multicolumn{2}{|l|}{ Complex } & $\mathrm{r}, \AA$ & $\theta$, & $\tau,{ }^{\circ}$ & Ref. \\
\hline \multicolumn{2}{|c|}{ galactose oxidase $Y 272$} & 1.91 & 127 & -72 & $(10)$ \\
\hline & Y495 & 2.56 & 105 & 53 & \\
\hline \multirow[t]{2}{*}{ Cu lactoferrin } & Y92 & 2.54 & 129 & -77 & $(12,13)$ \\
\hline & Y192 & 1.97 & 143 & .89 & \\
\hline \multicolumn{2}{|c|}{ [Cu(PMDT)cresol] } & 1.84 & 118 & 13 & (14) \\
\hline \multirow{2}{*}{\multicolumn{2}{|c|}{$\begin{array}{r}{\left[\mathrm{Cu}_{2}(\mathrm{dnc})_{2}\right] \text { phenol }} \\
\mathrm{S}-\mathrm{CH} 3 \text { phenol }\end{array}$}} & 1.95 & 121 & 46 & (15) \\
\hline & & 1.89 & 125 & -34 & \\
\hline
\end{tabular}



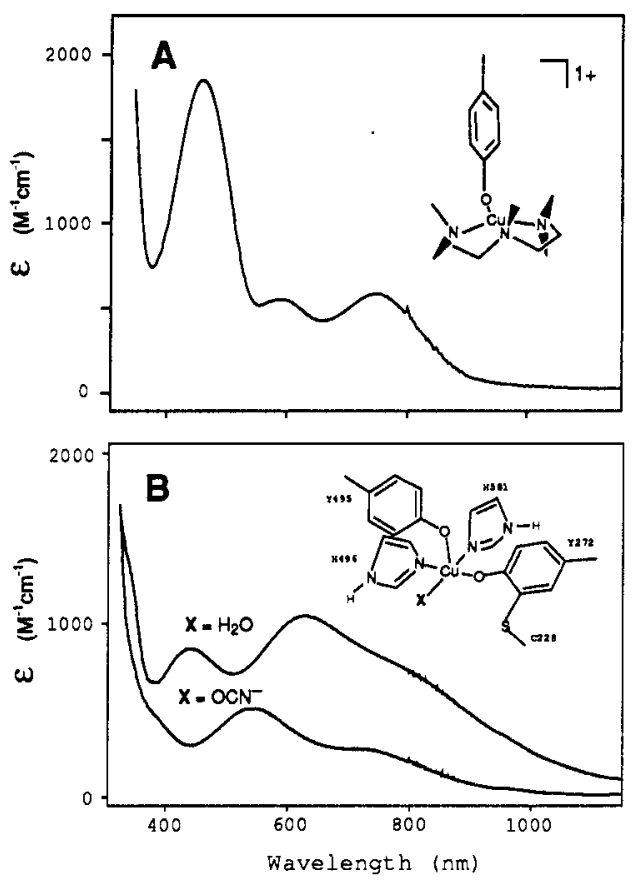

Fig. 3. Absorption spectra for $\mathrm{Cu}$ phenolate complexes. (A) [Cu(PMDT)cresol] $]^{+}$; (B) Inactive galactose oxidase.
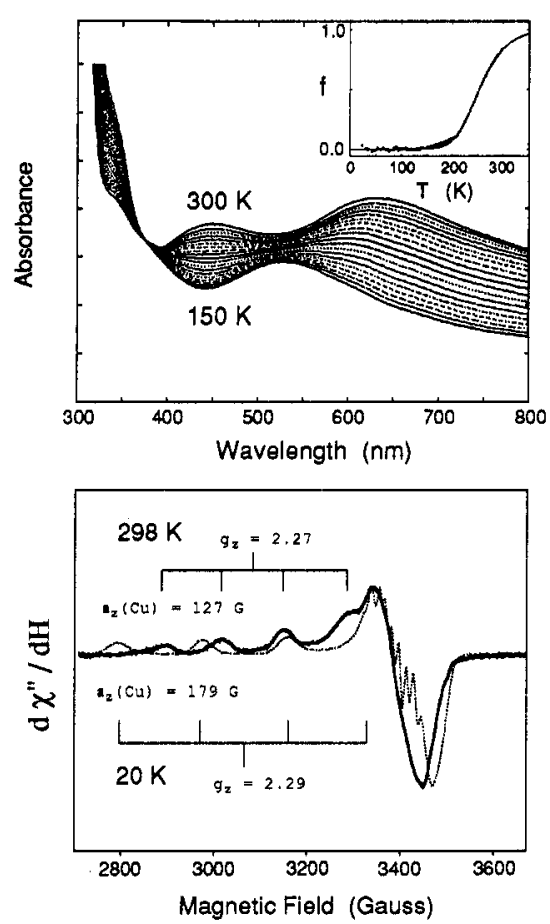

Fig. 4. Variable temperature absorption (Top) and EPR (Bottom) spectra for inactive galactose oxidase.Insert Conversion fraction (f) for the transition.

ligands on forming anion complexes [5,10]. On the other hand, spectroscopic investigations of anion interactions reveal that ligand binding results in a significant and fundamental transformation in the active site properties that is reflected in the optical absorption spectra [16,17]. Assignment of a strong absorption band near $450 \mathrm{~nm}$ in the electronic spectra of simple copper phenolate models (Fig. 3, A) as phenolate-to$\mathrm{Cu}$ (II) ligand-to-metal charge transfer (LMCT) [14] leads to analogous assignment of the corresponding feature in the spectrum of the radical-free $\mathrm{Cu}$ (II) protein (Fig. 3, B) as LMCT arising from the unmodified tyrosinate $Y 495[1,17,18]$. The relatively low intensity of the protein absorption is consistent with the relatively long bond distance $(r=2.56 \AA$ for the protein versus $1.84 \AA$ for the model $)$ and the distinct angular features of the protein complex in which the phenolate is shifted out of the equatorial ligand plane. This absorption disappears on binding anions, implying that the unmodified Y495 is displaced from the metal as a result of reorientation of the principal axis of the tetragonal ligand field along the metal-phenolate vector. This reorganization is expected to result in an increase in the basicity of the phenolate oxygen, and in fact anion binding leads to proton uptake by a base in the protein with $\mathrm{pK}_{\mathrm{a}}>9$, consistent with involvement of the Y495 phenolate [17]. Modulation of the basicity of this metal ligand by exogenous ligand interactions is expected to be important for substrate activation in catalysis $[1,17]$.

Even without exogenous ligand binding, the active site of galactose oxidase exhibits unusual structural instability. Simply varying the temperature from ambient $(300 \mathrm{~K})$ to cryogenic $(150 \mathrm{~K})$ leads to conversion of the resting enzyme from phenolate-bound to phenolate-free form (Fig. 4, Top) [17]. The transition is gradual, occurring over a temperature range of more than $100 \mathrm{~K}$ as is typical of molecular transitions involving local melting of macromolecular structure [19]. The transition also results in a reduction in the parallel component of the metal hyperfine interaction $\left(\mathrm{a}_{\|}(\mathrm{Cu})\right)$ and a decrease in the value of $\mathrm{g}_{\mathrm{z}}$ in the ground state EPR spectra reflecting an altered metal environment (Fig. 4, Bottom). The structural basis for this transition is essentially the same as described above for proton-coupled anion interactions. In this case, an internal proton transfer converts the ambient temperature aquo/phenolate complex into an isomeric hydroxide complex at low temperature in which the phenol is displaced and protonated, defining a proton transfer coordinate in the active site [19]. This isomerization of the active site structure reveals an important coupling between proton transfer involving two adjacent metal ligands and a change in geometry at copper, effectively describing a pseudorotation process in which the weak perturbation axis of the metal ion is reoriented by rearrangement of the ligands. At the physiological growth temperature of Fusarium sp. the transition is just completed indicating that this subtle instability of the protein complex is likely to be a biologically important adaptation shaping essential features of the reaction pathway. 


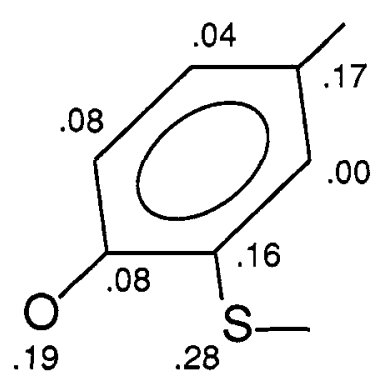

Fig. 5. Calculated distribution of the unpaired electron over the ground state $\pi$-system of the S-methyl substituted phenoxyl radical (Ref. (21)).

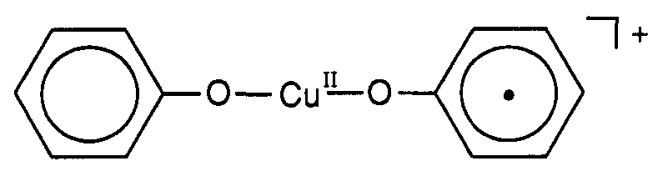

Fig.7. Mixed -valent bisphenolate model for the radical-Cu complex.

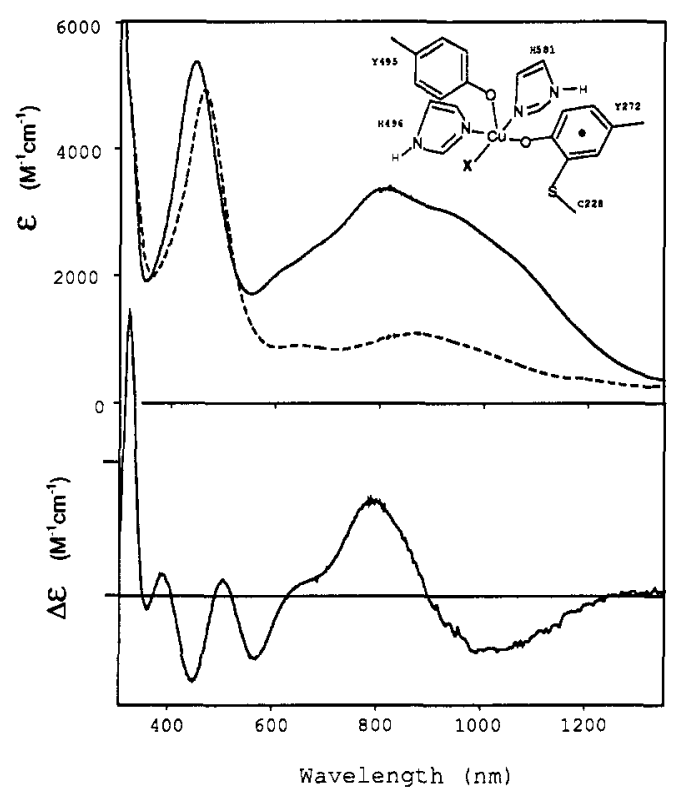

Fig. 6. Absorption (Top) and circular dichroism (Bottom) spectra for active galactose oxidase free radical-Cu complex. Ligand-free enzyme (solid line) or cyanate complex (broken line).

\section{THE FREE RADICAL SITE}

The simple $\mathrm{Cu}$ (II) coordination complex described above is catalytically inactive and requires redox activation to become competent for turnover. Activation involves oxidation of the protein to generate a free radical site that is electronically coupled to the metal center, forming a free radical coupled copper complex [1,4]. This radical is remarkably stable compared to radicals formed by radiation damage [4] and persists at ambient temperature with a half-life of nearly a week. By removing the metal and oxidizing the apo-enzyme, the radical site can be prepared for detailed independent characterization [20]. Optical absorption [20], EPR [20,21], and ENDOR [22] experiments have conclusively shown that the apoenzyme radical is localized on the Tyr-Cys dimer with little or no delocalization onto the stacked Trp residue or other groups. The g-tensor measured in high frequency EPR experiments is nearly identical to that of a simple thioether-substituted phenoxyl model [21]. Computational studies on the radical ground state lead to a predicted distribution of unpaired electron density (Fig. 5) having predominant contributions from the $\mathrm{p}_{z}$ orbitals on the phenoxyl oxygen and the thioether sulfur, mapping out the redox orbital on the free radical ligand and emphasizing the important role of the phenoxyl oxygen in ligand redox and metal interactions [21]. These calculations also underscore the importance of the thioether side chain in defining ground state properties. Thioether substitution leads to a decrease in redox potential of $0.5 \mathrm{~V}$ or more [14,15,23], suggesting one role of the Tyr-Cys dimer is to tune the redox potential of the tyrosine, making it more accessible for catalysis. Thioether substitution also specifically stabilizes a one-electron redox step, in contrast to quinones which are active as two electron redox units. In the quinone-copper active sites of the amine oxidases, the quino-cofactor $[24,25]$ serves as a two electron unit and the semiquinone radical appears to be formed only under unusual conditions [26].

\section{THE ACTIVE ENZYME RADICAL-COPPER COMPLEX}

The active enzyme (formed by either mild oxidation of the native state or reduction to the $\mathrm{Cu}(\mathrm{I})$ complex followed by $\mathrm{O}_{2}$ reoxidation [4]) has a very unusual optical absorption spectrum (Fig. 6, Top) associated with the presence of the Y272 free radical ligand. Based on X-ray absorption measurements, the oxidation state of metal ion remains unchanged in this process $[27,28]$, and electronic interactions between $\mathrm{Cu}(\mathrm{II})$ and the free radical ligand give rise to strong antiferromagnetic exchange coupling stabilizing a diamagnetic ground state [4]. These interactions are mediated by the direct sigma overlap of the phenoxyl $\pi$ system with the metal orbital containing the unpaired electron (Fig. 2). The optical spectrum is dominated by intense absorption near $445 \mathrm{~nm}$, with a second band in the NIR near $900 \mathrm{~nm}$. The true complexity of this spectrum is revealed in circular dichroism measurements (Fig. 6, Bottom) which resolve at least 9 components between 300 and $1200 \mathrm{~nm}$ [4]. The spectra are very sensitive to ligand interactions, and anion binding results in a dramatic 


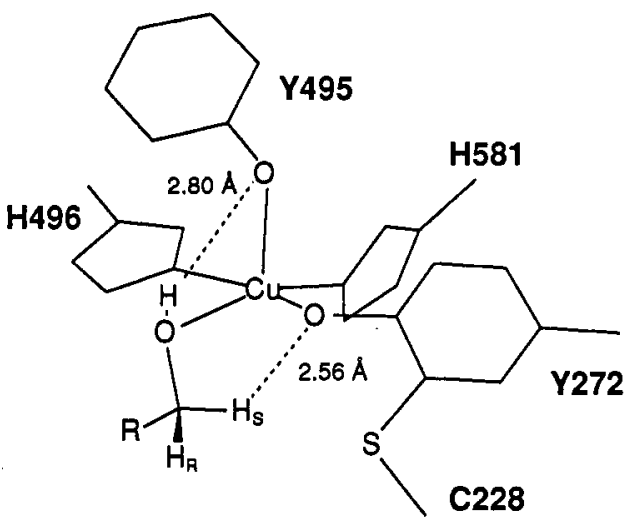

Fig. 8. Predicted structure of the substrate complex orienting both hydroxyl and methylene groups for reaction.

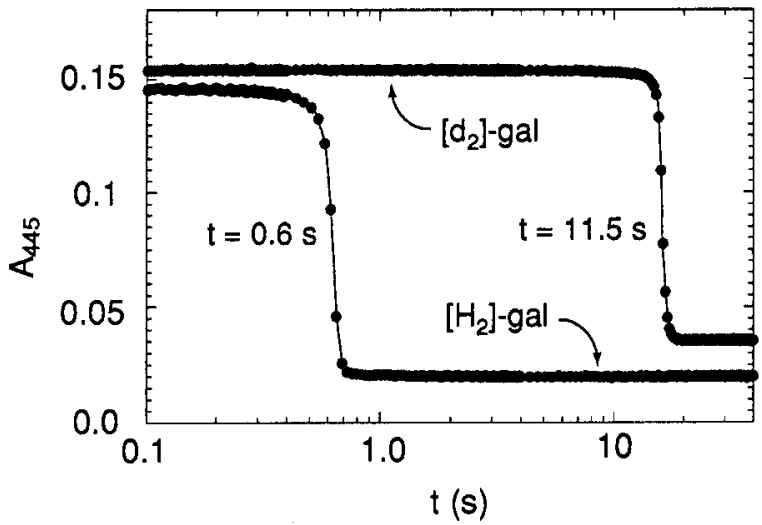

Fig. 9. Turnover clock reaction illustrating the dramatic $k_{\mathrm{H}} / k_{\mathrm{D}}$ isotope effect in galactose oxidase active site chemistry. $\left[\mathrm{d}_{2}\right]$-gal: 1-O-methyl-6,6'- $\left[{ }^{2} \mathrm{H}_{2}\right] \alpha$-D-galactopyranoside

decrease in intensity for the NIR absorption band. Resonance Raman excitation into the NIR band results in strong enhancement of both Y495 phenolate and Y272 phenoxyl modes in the ligand-free complex, while only phenoxyl modes are observed in anion complexes, where the electronic interaction between the two tyrosines is eliminated by displacement of Y495 $[7,29]$. The fact that both tyrosines contribute to the NIR absorption suggests assignment to a transition that spans both ligands, effectively a ligand-to-ligand charge transfer (LLCT) excitation, with smaller contributions from $\mathrm{Cu}(\mathrm{II}) d \rightarrow d$ and intraradical absorption.

The basic features of the active complex can be reduced to a simple picture of a mixed valent bisphenolate complex in which two (nearly) equivalent ligands in different oxidation states are bridged by a metal ion (Fig. 7). This essentially reverses the role of ligand and bridge in a conventional mixed valent complex. In this view, the complexity of the spectrum can be understood in terms of at least five major contributions: intraradical $\mathrm{n} \rightarrow \pi$ absorption; phenolate-to- $\mathrm{Cu}$ (II) LMCT; $\mathrm{Cu}$ (II)-to-phenoxyl metal-to-ligand charge transfer (MLCT); phenolate-to-phenoxyl LLCT; and metal ligand field $(d \rightarrow d)$ excitation. Electronic coupling between the unmodified tyrosinate and the radical ligand (reflected in the intensity of the LLCT absorption) may contribute to stabilizing the radical, since the mixed-valent description implies delocalization of the hole over the $\pi$ valence systems of both ligands. Thus, while the radical is strongly localized in the apoenzyme, there is evidence for delocalization over both tyrosine ligands in the $\mathrm{Cu}$ complex. In addition to these discrete contributions, electronic interactions in the complex will result in additional configurational excitations that would not occur for individual elements, making the 'hole' spectrum greater than the sum of its parts.

\section{RADICAL REDOX MECHANISM}

These geometric and electronic structural elements combine to form the efficient catalytic motif of the radical copper oxidase active site and determine the chemistry of this unique biological complex. The essential proton transfer and redox functions built into this active site define the key features of a catalytic mechanism for substrate oxidation. Although the structure of substrate complexes of galactose oxidase are unknown aside from model-building predictions [5,30], conservative replacement of the coordinated solvent hydroxyl with alcohol hydroxyl seems fully justified by the evidence for exogenous ligand interactions at the solvent site, with the additional stereochemical constraint of stereospecific pro-S methylene hydrogen abstraction in the oxidation step. This leads to a picture of proton transfer from the acidified hydroxyl of the coordinated alcohol to tyrosine $\mathrm{Y} 495$ phenolate triggering substrate activation [1,17]. The proton transfer coordinate (Fig. 8) involves a gap of less than $3 \AA$, and is likely mimicked in the temperature-dependent proton shift observed for the inactive enzyme (Fig. 4). Alkoxide coordinated to $\mathrm{Cu}$ (II) will be unstable toward inner sphere electron transfer to form an alkoxyl radical-Cu(I) intermediate, the spins of the alkoxyl and the phenoxyl being correlated via initial state coupling and spin selection rules. The substrate radical is further activated for methylene $\mathrm{C}-\mathrm{H}$ bond homolysis, with delocalization of the unpaired electron density of the alkoxyl radical resulting in a weaker binding potential along the $\mathrm{C}-\mathrm{H}$ stretch (the reaction coordinate for bond cleavage). The unpaired electron density in the $2 \mathrm{p}_{\mathrm{z}}$ orbital of the phenoxyl oxygen found in spin unrestricted calculations in both density functional (DMol DFT)[21] and Hartree-Fock theory [31] of the radical ground state is favorably orientated toward the substrate methylene facilitating hydrogen atom transfer. 


\section{ISOTOPE KINETICS - NEW EVIDENCE FOR HYDROGEN TRANSFER}

Isotope kinetics have been reported for galactose oxidase turnover with the isotopic substrate 1-O-methyl6,6 -di-[ $\left[{ }^{2} \mathrm{H}_{2}\right] \alpha$-D-galactopyranoside indicating a large $k_{\mathrm{H}} / k_{\mathrm{D}}$ kinetic isotope effect $(=8.7)$ at ambient temperature in the steady state [32]. With David Ballou (University of Michigan) we have extended these kinetic experiments to explore the isotope effects in the isolated substrate oxidation half-reaction, uncoupling the kinetic contribution of $\mathrm{O}_{2}$ reduction. Since $\mathrm{C}-\mathrm{H}$ bond cleavage occurs during substrate oxidation the majority of the kinetic isotope effect associated with deuterium-labelled substrate is expected to be expressed in this specific half reaction. The experimental measurement of this isotope effect requires stopped-flow rapid reaction techniques and high enzyme concentrations to directly monitor the reaction between active enzyme and reducing substrate. The progress of the reaction can be followed by measuring the disappearance of the UV-visible absorption of the active enzyme as the oxidized complex is reduced to the coloriess $\mathrm{Cu}(\mathrm{I})$ form. A dramatic isotope effect of nearly 20 is observed in this reaction, consistent with a hydrogen atom transfer step [33-36] in substrate oxidation. This isotope effect is also clearly illustrated in a turnover clock experiment shown in Fig. 9 which takes advantage of the extremely fast reaction with oxygen. Using a fixed concentration of dissolved oxygen to support turnover, the enzyme in the steady state of the reaction (early in the logarithmic timecourse displayed in Fig. 9) retains the full absorbance of the starting active complex, indicating that the $\mathrm{O}_{2}$ reoxidation step is fast compared to reduction by the organic substrate and is therefore not rate-limiting. The steady state reaction proceeds until the $\mathrm{O}_{2}$ initially dissolved in the sample is consumed at which point the remaining substrate rapidly reduces the complex, quenching the absorbance. The number of catalytic turnovers required to reach this point is fixed by the amount of dissolved oxygen and is thus the same for both the isotopic and non-isotopic reactions, allowing the substrate reduction rates to be directly compared using the 'turnover clock'. As shown in Fig. 9, the turnover clock runs nearly 20 -fold slower for the deuterium-labelled substrate for the non-isotopic sample. This dramatic kinetic isotope effect can be viewed as the signature of a hydrogen atom in flight in the transition state [33-36], providing strong evidence for a radical redox mechanism for substrate oxidation $[4,17]$.

\section{SUMMARY}

The active sites of the radical copper oxidases are designed to function as radical redox engines performing two-electron reactions with two distinct elements, a redox-active metal center and a free radical ligand. This separation of the redox centers requires the reaction to proceed in one-electron steps as a result of the inequivalence of the two sites. The ligands play a central role in this mechanism, participating to a much greater extent than is usually recognized for metalloenzyme complexes. The unmodified tyrosine Y495 appears to serve as a general base for catalysis, abstracting a proton from the coordinated substrate hydroxyl. The Tyr-Cys dimer Y272, on the other hand, is engineered for redox chemistry, with strong electronic coupling to the $\mathrm{Cu}$ ion. The perpendicular coordination mode of the redox-active tyrosine leads to covalent overlap

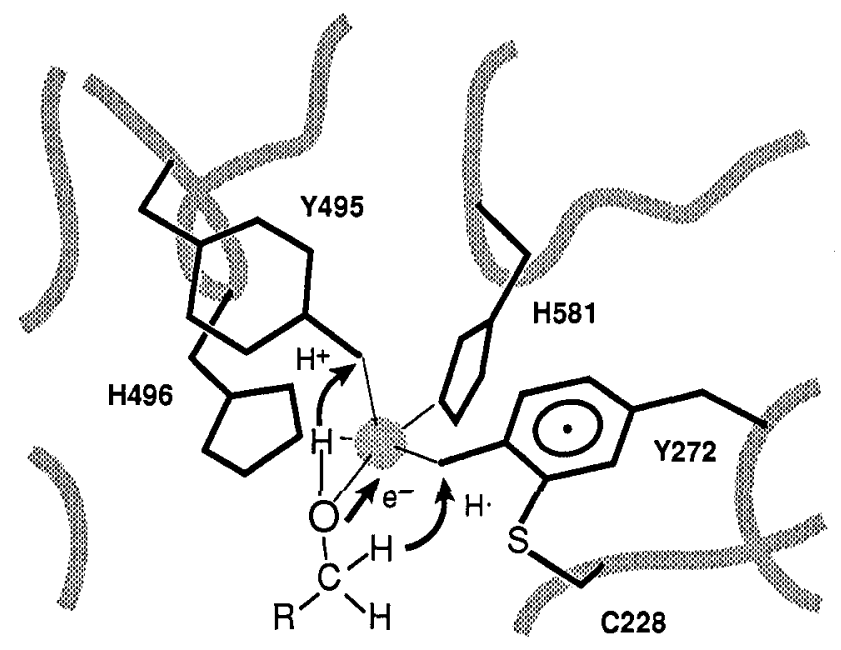

Fig. 10. Proposed substrate oxidation mechanism for galactose oxidase involving proton transfer, inner sphere electron transfer, and hydrogen atom transfer in the framework of the free radical-copper complex. (Based on Ref. (17).) 
between the metal and ligand redox orbitals that are likely to be important for the chemistry of this complex. Tyrosine ligands are thus involved in substrate oxidation in both proton and hydrogen transfer steps. The elementary redox chemistry of the active site includes inner sphere reduction of the metal ion and transfer of hydrogen to the phenoxyl ligand (Fig. 10). The orientation of the substrate in the active site is clearly critical for catalysis and this constraint may account for the strict regioselectivity of galactose oxidase. An analogous mechanism can be drawn for glyoxal oxidase, with the hydrated carbonyl of the substrate acetal replacing the alcohol hydroxyl as a metal ligand. In the second half reaction of the turnover cycle, reduction of $\mathrm{O}_{2}$ likely proceeds by essentially reversing these steps, with rapid two-electron reduction of dioxygen to form a $\mathrm{Cu}$ (II)hydroperoxy anion complex that dissociates on transfer of a proton from tyrosine Y495 phenol to the proximal oxygen of the coordinated product.

\section{WHY DO CHEMISTRY ONE ELECTRON AT A TIME?}

The question remains: What is the advantage of doing two-electron chemistry one electron at a time, using a radical redox reaction instead of more typical heterolytic mechanism? Free radical chemistry may provide an advantage in allowing rapid and indiscriminant utilization of a wider variety of reducing substrates supporting the most rapid formation of hydrogen peroxide. In addition, there may be a regulatory significance to the redox activation step characteristic of the radical copper oxidases. These enzymes can be either 'on' or 'off' depending on the redox state of the active site. In the extracellular environment in which these enzymes are designed to function, there is an advantage in an enzyme not always being 'on', and in the absence of the complex control circuits of intracellular metabolism regulation can be effectively achieved by balancing the degree of redox activation $[6,7]$.

\section{ACKNOWLEDGEMENTS}

This work has benefitted from the input of a large number of talented collaborators who have co-authored the literature from our lab. For recent work we would particularly like to thank Christopher A. Ekberg, Philip J. Kersten, Joann Sanders-Loehr and David P. Ballou. Support for this research from the National Institutes of Health (grant number GM 46749) is gratefully acknowledged.

\section{REFERENCES}

1. J.W. Whittaker, In Metal Ions in Biological Systems Vol. 30 (H. Sigel and A. Sigel, eds.), pp. 315-360. Marcel Dekker, Inc., New York (1994).

2 G. Avigad, C. Asensio, D. Amaral and B.L. Horecker Biochem. Biophys. Res. Commun 4, 474-477 (1961).

3. D.J. Kosman. In Copper Proteins and Copper Enzymes (R. Lontie, ed.) Vol. 2 pp. 1-26 (1984) CRC Press, Boca Raton.

4. M.M. Whittaker and J.W. Whittaker. J. Biol. Chem. 263, 6074-6080 (1988).

5. P.F. Knowles and N. Ito. Perspect. Bioinorg. Chem. 2, 207-244 (1993).

6. P.J. Kersten and T.K. Kirk. J. Bact. 169, 2195-2202 (1987).

7. M.M. Whittaker, P.J. Kersten, N. Nakamura, J. Sanders-Loehr, E.S. Schweizer and J.W. Whittaker. J. Biol. Chem. 271, 681-687 (1996).

8. P. Bork and R.F. Doolittle. J. Mol. Biol. 236, 1277-1282 (1994).

9. B. Silakowski, A. Pospiech, B. Neumann and H.U. Schairer J. Bact. 178, 6706-6713 (1996).

10. N. Ito, S.E.V. Phillips, C. Stevens, Z.B. Ogel, M.J. McPherson, J.N. Keen, K.D.S.Yadev, and P.F. Knowles. Nature (London) 350, 87-90 (1991).

11. M.M. Whittaker, V.L. DeVito, S.A. Asher, and J.W. Whittaker. J. Biol. Chem. 264, 7104-7106 (1989).

12. C.A. Smith, B.A. Anderson, H.M. Baker and E.N. Baker Biochem. 331, 4527-4533 (1992).

13. H.M. Baker, B.A. Anderson, A.M. Brodie, M.S. Shongwe, C.A. Smith and E.N. Baker Biochem. 35, 9007-9013 (1996).

14. M.M. Whittaker, Y.Y. Chuang and J.W. Whittaker. J. Am. Chem. Soc. 115, 10029-10035 (1993).

15. M.M. Whittaker, W.R. Duncan and J.W. Whittaker. Inorg. Chem. 35, 382-386 (1996).

16. J.W. Whittaker. In Bioinorganic Chemistry of Copper (K.D. Karlin and Z. Tyeklar, eds.) pp. 447-458. Chapman and Hall, New York (1993).

17. M.M. Whittaker and J.W. Whittaker. Biophys. J. 64, 762-772 (1993).

18. J.W. Whittaker. In Redox Active Amino Acids in Biology (J.P. Klinman, ed.) pp. 262-277. Academic Press, New York (1995).

19. J.W. Whittaker. J. Phys. Chem. B 101, 674-677 (1997).

20. M.M. Whittaker and J.W. Whittaker. J. Biol. Chem. 265, 9610-9613 (1990).

21. G.A. Gerfen, B. Bellew, R. Griffin, D. Singel, C.A. Ekberg, J.W. Whittaker. J. Phys. Chem. 100, 16739-16748 (1996).

22. G.T. Babcock, M.K. El-Deeb, P.O. Sandusky, M.M. Whittaker and J.W. Whittaker. J. Am. Chem. Soc. 114, $3727-3734$ (1992).

23. S. Itoh, K. Hirano, A. Furuta, M. Komatsu, Y. Ohshiro, A. Ishida, N. Takamuku and S. Suzuki. Chem. Lett. 2099-2102 (1993). 
24. J.P. Klinman and D. Mu. Annu. Rev. Biochem. 63, 299-344 (1994).

25. S.M. Janes, D. Mu, D. Wemmer, A.J. Smith, S. Kaur, D. Maltby, A.L. Burlingame and J.P. Klinman. Science 248, 981-987 (1990).

26. D.M. Dooley, M.A. McGuirl, D.E. Brown, P.N. Turowski, W.S. McIntire and P.F. Knowles. Nature (London) 349, $262-264$ (1991).

27. K. Clark, J.E. Penner-Hahn, M.M. Whittaker and J.W. Whittaker. Biochemistry 33, 12553-12557 (1994).

28. K. Clark, J.E. Penner-Hahn, M.M. Whittaker and J.W. Whittaker. J. Am. Chem. Soc. 112, 6433-6434 (1990).

29. M.L. McGlashin, D.D. Eads, T.G. Spiro and J.W. Whittaker. J. Phys. Chem. 99, 4918-4922 (1995).

30. R. Wachter and B.P. Branchaud. J. Am. Chem. Soc. 118, 2782-2789 (1996).

31. S. Itoh, S. Fukuzumi and S. Takayama. Inorg. Chem. 36, 1407- (1997).

32. J.J. Villafranca, J.C. Freeman and A. Kotchevar. In Bioinorganic Chemistry of Copper (K.D. Karlin and Z. Tyeklar, eds.) pp. 439-446. Chapman and Hall, New York (1993).

33. Y. Cha, C.J. Murray and J.P. Klinman. Science 243, 1325-1330 (1989).

34. B.J. Bahnson and J.P. Klinman. Meth. Enzymol. 249, 373-397 (1995).

35. J.P. Klinman. Trends. Biochem. Sci. 14, 368-373 (1989).

36. J.P. Klinman. Adv. Enzymol. Relat. Areas Mol. Biol. 46, 415-494 (1978). 